\title{
The role of factor $X a$ inhibitors in venous thromboembolism treatment
}

This article was published in the following Dove Press journal:

Vascular Health and Risk Management

30 January 2015

Number of times this article has been viewed

\author{
Katherine P Cabral' \\ Jack E Ansell ${ }^{2}$ \\ 'Department of Pharmacy Practice, \\ Albany College of Pharmacy and \\ Health Sciences, Albany, NY, USA; \\ ${ }^{2}$ Hofstra-North Shore/LIJ School \\ of Medicine, Hempstead, NY, USA
}

Correspondence: Katherine P Cabral Pharmacy Practice, Albany College of Pharmacy and Health Sciences, $106 \mathrm{New}$ Scotland Avenue, Albany, NY 12208, USA $\mathrm{Tel}+15186947187$

Fax + I 5186947382

Email katherine.cabral@acphs.edu
Abstract: Three factor Xa inhibitors have been studied in the treatment of venous thromboembolism, both for acute therapy and as extended therapy to prevent recurrent events. Rivaroxaban, apixaban, and edoxaban have all proven to be effective in Phase III clinical trials for this indication when compared to current standard of therapy with similar or less bleeding. Nevertheless, the agents all offer different pharmacological profiles, which have an impact on patient selection and potential advantages in clinical practice.

Keywords: anti-Xa inhibitors, apixaban, edoxaban, rivaroxaban, venous thromboembolism

\section{Introduction}

Anticoagulant therapy is the cornerstone of venous thromboembolism (VTE) treatment, and is typically provided with an initial parenteral agent, overlapping to an oral agent for maintenance therapy and prevention of recurrent VTE events. Patients are often treated with anticoagulant therapy for 3-6 months depending on the initiating event and other clinical factors; however, treatment may be extended longer to prevent recurrent VTE events. ${ }^{1}$ There has been little change to this fundamental approach to treating acute VTE until the recent introduction of the new, targetspecific oral anticoagulants: direct thrombin inhibitors and factor Xa inhibitors. Three oral factor Xa inhibitors, rivaroxaban, apixaban, and edoxaban, have all been studied in Phase III clinical trials for the treatment of VTE and are US Food and Drug Administration approved for this indication. The Xa inhibitors offer several advantages over traditional therapy with parenteral anticoagulant bridging to a vitamin $\mathrm{K}$ antagonist. This review will highlight the pharmacology of the oral $\mathrm{Xa}$ inhibitors, the available clinical trial data, and the potential advantages and role for their use in VTE treatment.

\section{Pharmacology of factor $\mathrm{Xa}$ inhibitors}

Factor Xa inhibitors are small molecules that selectively and reversibly bind to the active site of activated factor $\mathrm{X}(\mathrm{Xa})$, which blocks the interaction with its substrate in a rapid and competitive fashion, therefore inhibiting the final effects of thrombin generation. ${ }^{2}$ They inhibit both free factor $\mathrm{Xa}$ in solution and within a clot, and have no direct effect on platelet aggregation. Inhibiting the coagulation cascade in a targeted fashion has several advantages. Targeting factor $\mathrm{Xa}$, where the intrinsic and extrinsic pathways meet, inhibits thrombin generation from both pathways. In comparison to directly blocking thrombin, it is theorized that by inhibiting thrombin generation 
more proximally at factor Xa, the amplification of thrombin generation that occurs downstream may be prevented and therefore may require less drug for inhibition compared to the amount needed to directly inhibit at thrombin. ${ }^{2,3}$ Unlike thrombin, factor Xa also has minimal functions outside of the role of coagulation; therefore, negative effects as a consequence of inhibition may be limited. ${ }^{4,5}$

The Xa inhibitors exhibit linear pharmacokinetics and display predictable anticoagulant responses, thereby avoiding the need for routine monitoring. ${ }^{2}$ In general, all three of the oral factor-Xa inhibitors are rapidly absorbed, reaching a maximum concentration within approximately 3 hours (see Table 1). ${ }^{2-9}$ Minor differences in pharmacokinetics exist; for example, rivaroxaban has a high bioavailability which is dose dependent. The doses utilized for VTE treatment (15-20 mg) must be administered with food to maintain the high bioavailability, area under the curve (AUC), and maximum peak concentration (Cmax). ${ }^{5}$ Once in the plasma, rivaroxaban is highly protein-bound and has a low volume of distribution (Table 1), whereas apixaban and edoxaban's exposure and peak concentration are not affected by a fed state and therefore can be administered with or without food. ${ }^{7,10-12}$

Apixaban has a small volume of distribution, suggesting that it is primarily distributed in the blood and is $87 \%$ protein bound. ${ }^{2,7}$ In comparison, edoxaban has a high volume of distribution due to its relatively low protein binding (Table 1). ${ }^{2,13,14}$ Since it is minimally protein-bound, edoxaban may be able to be removed by dialysis.

All three agents are renally eliminated to varying degrees and have an elimination half-life much less than the vitamin $\mathrm{K}$ antagonists. Rivaroxaban has a dual mechanism of excretion, with one-third of the absorbed dose excreted unchanged in the urine and the remaining two-thirds of the dose excreted as inactive metabolites in both the urine and feces. ${ }^{2,6,15}$ Its short half-life of 5-9 hours is prolonged in elderly patients to 11-13 hours due to age-related renal-function decline. ${ }^{2,5,6}$ As a patient's renal function worsens, the clearance of rivaroxaban is reduced and the Cmax is increased. ${ }^{6}$ Therefore, use for VTE treatment should be avoided in patients with severe renal impairment (creatinine clearance $[\mathrm{CrCl}]<30 \mathrm{~mL} / \mathrm{min}$ ) as these patients were also excluded from the clinical trials. ${ }^{6,15}$ Apixaban is minimally excreted by the kidneys - approximately $25 \%$ - with the remainder excreted by the hepatobiliary route in the feces. As renal function declines, exposure to apixaban may increase and patients with a $\mathrm{CrCl}<30 \mathrm{~mL} / \mathrm{min}$ were also excluded from clinical trials. Caution may be warranted in this patient population; however, the medication is now approved for use in patients with severe end-stage kidney disease receiving hemodialysis. ${ }^{7}$ Edoxaban is eliminated via multiple pathways with $35 \%$ eliminated in the urine, yet over $70 \%$ of the edoxaban dose is excreted unchanged. ${ }^{2,8,16}$

All three factor Xa inhibitors are partially metabolized through the CYP450 system to some extent and are P-glycoprotein (P-gp) substrates, which carries implications for potential drug interactions and will be discussed later in this paper (Table 1).

\section{Phase III clinical trials in acute VTE treatment}

All three factor Xa inhibitors have been studied for the acute treatment of VTE, either as separate deep vein thrombosis

Table I Factor-Xa inhibitor's pharmacokinetic and pharmacodynamic characteristics

\begin{tabular}{|c|c|c|c|}
\hline & Rivaroxaban & Apixaban & Edoxaban \\
\hline VTE dose & I5 mg BID $\times 3$ weeks, then $20 \mathrm{mg}$ once daily & $10 \mathrm{mg}$ BID $\times 7$ days, then $5 \mathrm{mg}$ BID & $\begin{array}{l}60 \mathrm{mg} \text { QD after 7-10 days } \\
\text { heparin }\end{array}$ \\
\hline Renal dose adjustment & Yes, $\mathrm{CrCl}<30 \mathrm{~mL} / \mathrm{min}$ & Yes, $\mathrm{CrCl}<25 \mathrm{~mL} / \mathrm{min}$ or $\mathrm{Scr}>2.5$ & $\begin{array}{l}\text { Assumed } 50 \% \text { reduction if } \\
\mathrm{CrCl}<50 \mathrm{~mL} / \mathrm{min}\end{array}$ \\
\hline $\operatorname{Tmax}(\mathrm{h})$ & $2-4$ & $3-4$ & $\mathrm{I}-2$ \\
\hline VD (L) & 50 & $\sim 23^{*}$ & $>300$ \\
\hline Half-life $(h)$ & $5-9$ & $9-14$ & $10-14$ \\
\hline Bioavailability & $>80 \%$ & $>50 \%$ & $62 \%$ \\
\hline Protein binding & $92 \%-95 \%$ & $87 \%$ & $40 \%-59 \%$ \\
\hline Metabolism & CYP3A4, CYP2J2 & CYP3A4 & CYP3A4 \\
\hline Elimination & $33 \%$ renal & $25 \%$ renal & $35 \%$ renal \\
\hline Effects of food & Cmax and AUC increased; take with food & Cmax and AUC unchanged & Cmax and $A U C$ unchanged \\
\hline CYP3A4 substrate & Yes & Yes & Yes \\
\hline P-gp substrate & Yes & Yes & Yes \\
\hline
\end{tabular}

Notes: *VD $=0.3 \mathrm{~L} / \mathrm{kg}$ and assuming a $75 \mathrm{~kg}$ patient. The HOKUSAI-VTE trial ${ }^{20}$ reduced dose by $50 \%$ in those patients with a $\mathrm{CrCl}$ of 30 to $50 \mathrm{~mL} / \mathrm{min}$, or a body weight $\leq 60 \mathrm{~kg}$, or in patients receiving concomitant treatment with potent P-gP inhibitor.

Abbreviations: AUC, area under the curve; BID, twice daily; Cmax, maximum peak concentration; CrCl, creatinine clearance; CYP, cytochrome P450; h, hours; min, minutes; P-gP, P-glycoprotein; QD, every day; Scr, serum creatine; Tmax, time to maximum concentration; VD, volume of distribution; VTE, venous thromboembolism. 
(DVT) and pulmonary embolism (PE) trials, or together. The EINSTEIN investigators studied the use of rivaroxaban as initial treatment for both acute DVT and PE. ${ }^{17,18}$ The Acute DVT study was a randomized, open-label study comparing rivaroxaban $15 \mathrm{mg}$ twice daily (BID) for 3 weeks, then 20 mg daily, to standard therapy of enoxaparin bridged to a vitamin $\mathrm{K}$ antagonist in 3,429 patients with acute, symptomatic, objectively confirmed proximal DVT. ${ }^{17}$ Randomization and rivaroxaban treatment started on day 1 without a heparin lead-in. It was an event driven, noninferiority study with $62 \%$ of patients treated for 6 months. The primary efficacy outcome was symptomatic recurrent VTE, which was experienced by $2.1 \%$ of patients in the rivaroxaban group compared to $3.0 \%$ of patients receiving standard therapy (hazard ratio [HR] 0.68 ; 95\% confidence interval $[\mathrm{CI}] 0.44-1.04 ; P<0.001$ for noninferiority). There was no statistically significant difference in the primary safety outcome of first major or clinically relevant nonmajor bleed (Table 2). Patients in the standard therapy group were on enoxaparin for a median of 8 days (interquartile range 6-11 days), then bridged to warfarin or acenocoumarol with a goal International Normalized Ratio (INR) 2-3, and spent $57.7 \%$ of time in therapeutic range (TTR). Therefore, it was determined that rivaroxaban was noninferior to standard therapy for the treatment of acute DVT.

A similar study was conducted by the same investigators looking at treatment of acute PE. ${ }^{18}$ EINSTEIN PE was a randomized, open-label study in 4,832 patients with acute symptomatic PE with or without DVT randomized to rivaroxaban $15 \mathrm{mg}$ BID for 3 weeks then $20 \mathrm{mg}$ once daily, or standard therapy of enoxaparin bridging to warfarin. Once again, rivaroxaban was initiated without a heparin lead-in. Twenty-five percent of patients had a concomitant DVT and the majority of patients were treated for 6-12 months. Rivaroxaban was found to be noninferior to standard therapy in reducing the rate of recurrent VTE $(2.1 \%$ versus $1.8 \%$; HR 1.12; 95\% CI $0.75-1.68 ; P=0.003$ for noninferiority) with a similar rate of major or clinically relevant nonmajor bleeding during treatment (10.3\% versus $11.4 \%$; HR 0.90 ; 95\% CI 0.76-1.07; $P=0.23$ ), although major bleeding was significantly reduced (1.1\% versus $2.2 \%$; HR $0.49 ; 95 \%$ CI $0.31-0.79 ; P=0.003)$. The TTR for patients on warfarin was $62.7 \%$ and the mean study duration was 9 months.

Apixaban has been studied for the treatment of acute VTE in the AMPLIFY study: a randomized, double-blinded, noninferiority trial. ${ }^{19}$ A total of 5,395 patients with an acute DVT, $\mathrm{PE}$, or both were included and randomized to receive either apixaban $10 \mathrm{mg}$ BID for 7 days then $5 \mathrm{mg}$ BID for 6 months (without a heparin lead-in phase), or standard therapy with enoxaparin bridging to warfarin, with a goal INR 2-3, for 6 months. The majority of patients $(65 \%)$ had a DVT only with $89.8 \%$ being unprovoked and $25 \%$ were treated for a PE only. The incidence of recurrent symptomatic VTE or death related to VTE, which was the primary efficacy outcome, was met in $2.3 \%$ of those patients receiving apixaban compared to $2.7 \%$ in the conventional group, meeting noninferiority criteria (Table 2). Interestingly, major bleeding, the primary safety outcome, was significantly less in the apixaban group compared to the conventional group $(0.6 \%$ versus $1.8 \%$;

Table 2 Phase III clinical trial results for the treatment of acute venous thromboembolism

\begin{tabular}{|c|c|c|c|c|}
\hline Phase III trials & Major outcomes & $\begin{array}{l}\text { Oral Xa inhibitor } \\
\text { (\% of events) }\end{array}$ & $\begin{array}{l}\text { Standard therapy } \\
\text { (\% of events) }\end{array}$ & $\begin{array}{l}\text { Hazard ratio, } 95 \% \text { confidence } \\
\text { interval }\end{array}$ \\
\hline \multicolumn{5}{|l|}{ Acute VTE treatment } \\
\hline \multirow[t]{2}{*}{ EINSTEIN-DVT'17 } & Symptomatic recurrent VTE & $2.1 \%$ & $3.0 \%$ & HR 0.68 (95\% Cl 0.44-I.04); $P<0.00$ I \\
\hline & Ist M/CRNMB & $8.1 \%$ & $8.1 \%$ & HR 0.97 (95\% Cl 0.76-I.22); $P=0.77$ \\
\hline \multirow[t]{2}{*}{ EINSTEIN-PE ${ }^{18}$} & Recurrent VTE & $2.1 \%$ & $1.8 \%$ & HR I.I2 (95\% Cl 0.75-I.68); $P=0.003$ \\
\hline & Ist M/CRNMB & $10.3 \%$ & $11.4 \%$ & HR 0.90 (95\% Cl 0.76-I.07); $P=0.23$ \\
\hline \multirow[t]{2}{*}{ AMPLIFY'19 } & $\begin{array}{l}\text { Ist recurrent VTE or VTE-related } \\
\text { death }\end{array}$ & $2.3 \%$ & $2.7 \%$ & HR 0.84 (95\% Cl 0.6-I.8); $P<0.001$ \\
\hline & Major bleed & $0.6 \%$ & $1.8 \%$ & HR 0.3 I (95\% Cl 0.17-0.55); $P<0.00$ I \\
\hline \multirow[t]{2}{*}{ HOKUSAI-VTE ${ }^{20}$} & Symptomatic recurrent VTE & $3.2 \%$ & $3.5 \%$ & HR 0.89 (95\% Cl 0.70-I.13); $P<0.001$ \\
\hline & Clinically relevant bleeding & $8.5 \%$ & $10.3 \%$ & HR $0.8 I$ (95\% Cl 0.7I-0.94); $P=0.004$ \\
\hline \multicolumn{5}{|c|}{ Extended VTE treatment } \\
\hline \multirow[t]{2}{*}{ EINSTEIN-Ext ${ }^{17}$} & Symptomatic recurrent VTE & $1.3 \%$ & $7.1 \%$ & HR 0.18 (95\% Cl 0.09-0.39); $P<0.00$ I \\
\hline & Ist M/CRNMB & $6.0 \%$ & $\mathrm{I} .2 \%$ (placebo) & HR 5.19 (95\% Cl 2.3-II.7); $P<0.00 \mid$ \\
\hline \multirow[t]{4}{*}{ AMPLIFY-Ext ${ }^{21, *}$} & Recurrent VTE/death from any & $4.2 \%(5 \mathrm{mg})$ & $11.6 \%$ (placebo) & HR $0.36(95 \% \mathrm{Cl} 0.25-0.53) ; P<0.00 \mathrm{I}$ \\
\hline & cause & $3.8 \%(2.5 \mathrm{mg})$ & & HR 0.33 (95\% Cl 0.22-0.48); $P<0.00$ I \\
\hline & Major bleeding & $0.1 \%(5 \mathrm{mg})$ & $0.5 \%$ (placebo) & HR 0.25 (95\% Cl 0.03-2.24); NS \\
\hline & & $0.2 \%(2.5 \mathrm{mg})$ & & HR 0.49 (95\% Cl 0.09-2.64); NS \\
\hline
\end{tabular}

Notes: "This study included two doses of $\mathrm{Xa}$ inhibitor and only one placebo. Data from. ${ }^{17-20,21}$

Abbreviations: $\mathrm{Cl}$, confidence interval; HR, hazard ratio; M/CRNMB, major or clinically relevant nonmajor bleeding; VTE, venous thromboembolism; NS, not significant. 
HR $0.31 ; 95 \%$ CI $0.17-0.55 ; P<0.001)$. This trend was also seen in the secondary endpoint of major bleeding or clinically relevant nonmajor bleeding: occurring $4.3 \%$ in the apixaban group versus $9.7 \%$ in conventional therapy, $P<0.001$. In the conventional group, the median duration of the enoxaparin bridge was 6.5 days (interquartile range 5-8 days) and the TTR for warfarin was $61 \%$. Efficacy and safety endpoint results were found to be consistent in those study centers where warfarin's mean TTR exceeded 68\%.

Edoxaban has been studied for the treatment of VTE in a randomized, double-blinded trial: HOKUSAI-VTE. ${ }^{20}$ The study required a heparin lead-in for the edoxaban arm (enoxaparin or unfractionated heparin) for at least 5 days followed by edoxaban $60 \mathrm{mg}$ once daily compared to a heparin bridge over to therapeutic warfarin, INR 2-3, in patients with DVT, $\mathrm{PE}$, or both. ${ }^{20}$ Patients receiving edoxaban were given a reduced dose of $30 \mathrm{mg}$ once daily if they had a $\mathrm{CrCl}$ of $30-50 \mathrm{~mL} / \mathrm{min}$, weighed $60 \mathrm{~kg}$ or less, or were receiving concomitant treatment with a potent $\mathrm{P}$-gp inhibitor. Patients were evaluated after 12 months regardless of the duration of treatment the patient received. About two-thirds of the overall 8,240 patients were treated for a DVT only. Edoxaban was determined to be noninferior to warfarin in preventing the primary efficacy outcome: incidence of symptomatic recurrent VTE (3.2\% of edoxaban versus $3.5 \%$ of warfarin patients; HR 0.89 ; 95\% CI 0.70-1.13; $P<0.001$ ) (Table 2 ). The primary safety outcome of clinically relevant bleeding was found in $8.5 \%$ of patients receiving edoxaban and $10.3 \%$ of those receiving warfarin (HR 0.81 ; $95 \%$ CI $0.71-0.94 ; P=0.004)$, meeting superiority criteria. This decreased trend in bleeding was also observed amongst the $17 \%$ of patients who received the reduced $30 \mathrm{mg}$ dose. Patients receiving warfarin were in range $63.5 \%$ of time.

\section{Phase III clinical trials in extended VTE treatment}

In addition to acute VTE treatment, rivaroxaban and apixaban were also studied as extended therapy to prevent further recurrent events. The EINSTEIN investigators studied rivaroxaban for the extended or continued treatment of VTE in the EINSTEIN-Extension trial. ${ }^{17}$ This was an eventdriven, superiority, double-blinded study that investigated 1,188 patients with a confirmed symptomatic VTE who had been treated for 6 or 12 months with rivaroxaban or a vitamin $\mathrm{K}$ antagonist and then randomized to receive continued therapy with rivaroxaban $20 \mathrm{mg}$ daily or placebo for another 6 or 12 months. Recurrent VTE occurred in $1.3 \%$ of patients receiving rivaroxaban compared to $7.1 \%$ of those on placebo (HR 0.18; 95\% CI 0.09-0.39; P<0.001), resulting in an $82 \%$ relative risk reduction (Table 2 ). The primary safety endpoint of first major or clinically relevant nonmajor bleeding occurred in $6.0 \%$ of those on rivaroxaban compared to $1.2 \%$ in the placebo group (HR 5.19; 95\% CI 2.3-11.7; $P<0.001)$ with the majority of the events being hematuria, epistaxis, or rectal or skin bleeding. There was no significant difference in major bleeding rates.

Similar to rivaroxaban, apixaban was also studied as extended treatment of VTE in the AMPLIFY-EXT trial. ${ }^{21}$ This randomized, double-blind study compared apixaban $5 \mathrm{mg}$ BID, $2.5 \mathrm{mg}$ BID, or placebo in 2,482 patients who completed 6-12 months of initial VTE treatment. Two-thirds of patients were treated for a DVT and one-third for a PE, with majority (91\%) of events being unprovoked. After the 1-year study period, the primary efficacy outcome of recurrent VTE or death from any cause occurred in $4.2 \%$ of patients receiving apixaban $5 \mathrm{mg}$ (HR 0.36 ; 95\% CI $0.25-0.53 ; P<0.001$ ), 3.8\% of patients receiving apixaban $2.5 \mathrm{mg}$ (HR 0.33 ; 95\% CI $0.22-0.48$; $P<0.001$ ), and $11.6 \%$ of patients receiving placebo (Table 2 ). Both doses of apixaban resulted in significantly less recurrent VTE or deaths related to VTE compared to placebo $(1.7 \%$ [5 mg] versus $1.7 \%$ [2.5 mg] versus $8.8 \%$ [placebo]). There was no significant difference in the primary safety endpoint of major bleeding, and clinically relevant nonmajor bleeding was increased in the apixaban $5 \mathrm{mg}$ dose $(4.2 \%$; HR 1.82 ; 95\% CI 1.05-3.18) compared to the $2.5 \mathrm{mg}$ (3.0\%; HR 1.29; 95\% CI $0.72-2.33)$ and placebo (2.3\%).

\section{Oral factor-Xa inhibitors: potential clinical advantages and their role in VTE treatment}

As the Phase III trials suggest, the new oral Xa inhibitors are as effective as standard-of-care therapy and have similar, or less in the case of apixaban and edoxaban, major bleeding events compared with standard therapy. Nevertheless, providers should be cognizant of the details of the Phase III clinical trials, as well as their clinical pharmacology and clinical implications, when determining individualized patient selection of these agents for the treatment of a VTE event.

One advantage is the lack of the need for a heparin lead-in phase, at least for rivaroxaban and apixaban. Even though all of the new oral Xa inhibitors have a relatively quick onset of action, reaching peak concentrations within approximately 3 hours, the edoxaban investigators opted to use a heparin lead-in for all patients. Therefore, at this point, we do not have data available to determine if the efficacy and safety of starting edoxaban immediately at VTE presentation would yield the same outcomes. 
Exclusion criteria for the Phase III trials were extensive and warrant attention. Several patient populations were not studied which may limit the generalizability of the trials. There were very few patients above 75 years of age included in these trials; in fact, in AMPLIFY-EXT, only $15 \%$ of patients were older than 75 years of age. ${ }^{21}$ Patients with poor renal function $(\mathrm{CrCl}<30 \mathrm{~mL} / \mathrm{min})$ were excluded from the trials. HOKUSAI-VTE did provide a dose reduction for those patients with a $\mathrm{CrCl}$ of $30-50 \mathrm{~mL} / \mathrm{min}$, but the majority of patients in the Phase III trials had good renal function. The studies included a very small minority of patients that had thrombophilic conditions or cancer. In the EINSTEIN-PE trial, only about $5 \%$ of patients had a known thrombophilic condition. ${ }^{18}$ Additionally, very few patients had body weights under $60 \mathrm{~kg}$. The majority of patients were a mean of $70-80 \mathrm{~kg}$; therefore, minimal data exists in patients that are severely under- or overweight. Caution is warranted when extrapolating study results to these patient populations (particularly as several of these comorbidities increase the risk or are present in those with VTE) and when determining individualized therapy regimens for any patients that fit such populations or other exclusion criteria from the trials.

Another exclusion criterion from the trials was concomitant medications that would interact with the factor Xa inhibitors. Although less drug interactions exist than with warfarin, all three of the Xa inhibitors discussed are CYP3A4 substrates, as well as P-gp substrates, which imply they have the potential to interact with many medications that are inhibitors or inducers of the enzymes. When given concomitantly with an oral Xa inhibitor, strong CYP3A4 and P-gp inhibitors, such as azole antifungals or HIV-protease inhibitors, have the propensity to increase the anticoagulant's concentration. We have seen this interaction with rivaroxaban result in a more than twofold increase in rivaroxaban's mean AUC and Cmax. ${ }^{6,22}$ Additionally, coadministration with oral ketoconazole once daily led to a twofold increase in apixaban's mean AUC and 1.6-fold increase in its mean Cmax. ${ }^{23}$ Medications that moderately inhibit CYP3A4 or $\mathrm{P}$-gp are expected to increase the plasma concentrations of the oral Xa inhibitors, but to a lesser extent. Therefore, it is recommended that strong CYP3A4 or P-gp inhibitors not be used in combination with the oral anti-Xa inhibitors to avoid increased bleeding risk. Conversely, the combination of the anticoagulants with strong CYP3A4 or P-gp inducers, such as rifampicin, will lead to decreases in plasma concentrations of the Xa inhibitors. In particular, when rifampicin was given with rivaroxaban, it led to a $50 \%$ decrease in rivaroxaban's plasma concentration. ${ }^{6}$ Likewise, apixaban combined with rifampin led to a $54 \%$ decrease in apixaban's mean AUC and a $42 \%$ decrease in Cmax. ${ }^{7}$ Such decreases in exposure may translate into decreased efficacy and therefore concomitant administration should be avoided. In fact, patients that were receiving strong CYP3A4 inhibitors or inducers were excluded from the EINSTEIN trials, as well as the AMPLIFY trials. Edoxaban undergoes CYP450 metabolism to a lesser degree than rivaroxaban and apixaban; nevertheless, it does interact with strong P-gp inhibitors. Based on pharmacokinetic studies, when edoxaban was administered with strong P-gp inhibitors, quinidine, verapamil, and dronedarone, it led to an increase in edoxaban's maximum concentration and exposure. ${ }^{24}$ Dose adjustments for such combinations can be anticipated. In fact, the HOKUSAI-VTE study reduced the dose if patients were on potent P-gp inhibitors, and the Phase III study of edoxaban for stroke prevention in atrial fibrillation, the ENGAGE AF-TIMI 48 trial, mandated a protocol-directed $50 \%$ reduction of the edoxaban dose for those patients receiving verapamil or quinidine. ${ }^{25}$

As with all anticoagulants, vigilance with concomitant use of antiplatelets is warranted to avoid potentiation of antithrombotic effects and increased bleeding risks. All of the Phase III studies discussed here excluded dual antiplatelet therapy, as well as limited aspirin use to either doses $<100 \mathrm{mg}$ or $>165 \mathrm{mg}$. The EINSTEIN trial was the only trial that, although discouraged, did allow dual antiplatelet therapy of clopidogrel $75 \mathrm{mg}$ daily and aspirin $<100 \mathrm{mg}$ daily. Since minimal information therefore exists with the oral Xa inhibitors in combination with the various antiplatelet medications or high dose aspirin, close monitoring is recommended to minimize bleeding risks.

Other advantages of the oral Xa inhibitors that are worth considering when determining individualized patient treatment include the administration schedule and adherence. Rivaroxaban is given twice daily for 3 weeks, then once daily; apixaban is given twice daily with a dose change after 7 days; whereas edoxaban is a once daily medication. Since the three agents demonstrate linear, predictable therapeutic effects with a fixed dose, none of the agents require routine monitoring for anticoagulant effects, compared with warfarin's INR measurement. ${ }^{2}$ The absence of therapeutic monitoring could be viewed as an advantage to improve patients quality of life compared to frequent INR monitoring with warfarin, as well as decreasing the healthcare provider's workload. However, it also results in decreased opportunities for patient education, as well as decreased ability to tailor patient-specific therapy and to potentially detect issues early on, such as nonadherence, bleeding, or thrombosis risks. Adherence must be closely evaluated and discussed with the patient 
prior to oral anticoagulant agent selection, provided that the oral Xa inhibitors have a short half- life and nonadherence will quickly put the patient at increased risk of developing recurrent thrombosis.

In the absence of routine monitoring, the therapeutic effects of the oral Xa inhibitors at this point are not able to be reversed. Their short half-lives, compared to warfarin's, do provide some assurance that the drug concentrations will decline rapidly when therapy is discontinued in patients with normal renal function. However, reversibility in emergent situations, such as serious bleeding, trauma, or emergent surgery, is unclear. Limited animal studies have suggested that rivaroxaban can be reversed by infusion of recombinant factor VIIa or prothrombin complex concentrates (PCC). ${ }^{26}$ In addition, a study completed in 12 human subjects demonstrated that 4-factor PCC reverses the effects of rivaroxaban, determined by normalization of the prothrombin time and endogenous thrombin potential immediately after the dose was infused. ${ }^{27}$ There is also an ongoing Phase I trial investigating 4-factor PCC in patients treated with edoxaban. ${ }^{28}$ There is a factor Xa-specific antidote in development, andexanet alfa, which has shown favorable effects in Phase II trials. ${ }^{29}$ It is a recombinant, modified, specific factor Xa protein that targets and binds to direct and indirect factor Xa inhibitors in the blood, thereby inhibiting further binding to factor Xa, and allowing restoration of hemostasis. ${ }^{29,30}$ More evidence surrounding this antidote, and others, is forthcoming.

\section{Conclusion}

The oral factor Xa inhibitors, rivaroxaban, apixaban, and edoxaban, show promise as effective alternatives to the standard therapy for VTE treatment. The clinical trial results show noninferiority to standard therapy in acute treatment with similar or less bleeding. In addition, they may have a role in extended therapy to further prevent recurrent VTE events. As no head-to-head trials exist, one must be cautious in comparing and interpreting differences in the Phase III clinical trials; however, a recent, indirect comparison of the three agents showed no statistically significant difference in the risk of recurrent VTE or all-cause mortality between the agents, but apixaban appeared to be associated with lower risk of bleeding compared to the other agents. ${ }^{31}$ Differences in the clinical trials, including methodology and excluded patient populations, must be known when determining generalizability to the real-world population. In addition, vigilance to different pharmacological profiles and clinical implications is warranted when determining individualized patient care and agent selection of these new anticoagulants.

\section{Disclosure}

Jack Ansell discloses the following: consultant to Bristol Myers Squibb, Pfizer, Janssen, Boehringer Ingelheim, Daiichi Sankyo, Perosphere; speakers bureau for Boehringer Ingelheim; equity in Perosphere. Katherine Cabral reports no conflicts of interest in this work.

\section{References}

1. Kearon C, Akl EA, Comerota AJ, et al. Antithrombotic therapy for VTE disease: Antithrombotic Therapy and Prevention of Thrombosis, 9th ed: American College of Chest Physicians EvidenceBased Clinical Practice Guidelines. Chest. 2012;141(Supp1 2):e419S-e494S.

2. Eriksson BI, Quinlan DJ, Weitz JI. Comparative pharmacodynamics and pharmacokinetics of oral direct thrombin and factor xa inhibitors in development. Clin Pharmacokinet. 2009;48:1-22.

3. Alexander JH, Singh KP. Inhibition of Factor Xa: A potential target for the development of new anticoagulants. Am J Cardiovasc Drugs. 2005;5(5):279-290.

4. Rai R, Sprengeler PA, Elrod KC, Young WB. Perspectives on factor Xa inhibition. Curr Med Chem. 2001;8:101-119.

5. Kubitza D, Becka M, Wensing G, Voith B, Zuehlsdorf M. Safety, pharmacodynamics, and pharmacokinetics of BAY 59-7939 - an oral, direct Factor Xa inhibitor - after multiple dosing in healthy male subjects. Eur J Clin Pharmacol. 2005;61:873-880.

6. Janssen Pharmaceuticals, Inc. Xarelto ${ }^{\circledR}$ : Highlights of Prescribing Information. Janssen Pharmaceuticals, Inc.; 2011. Available from: http:// www.xareltohcp.com/sites/default/files/pdf/xarelto_0.pdf\#zoom=100. Accessed March 28, 2014.

7. Bristol-Myers Squibb. Eliquis: Highlights of Prescribing Information. Bristol-Myers Squibb; 2014. Available from: packageinserts.bms.com/ pi/pi_eliquis.pdf. Accessed October 19, 2014.

8. Ogata K, Mendell-Harary J, Tachibana M, et al. Clinical safety, tolerability, pharmacokinetics, and pharmacodynamics of the novel factor Xa inhibitor edoxaban in healthy volunteers. J Clin Pharmacol. 2010;50(7):743-753.

9. Matsushima N, Lee F, Sato T, Weiss D, Mendell J. Bioavailability and safety of the factor Xa inhibitor edoxaban and the effects of quinidine in healthy subjects. Clinical Pharmacology in Drug Development. 2013;2: 358-366.

10. Frost C, Wang J, Nepal S, et al. Apixaban, an oral, direct factor Xa inhibitor: single-dose safety, pharmacokinetics, pharmacodynamics and food effect in healthy subjects. Br J Clin Pharmacol. 2013;75(2):476-487.

11. Frost C, Yu Z, Shenker A, et al. Food does not affect the pharmacokinetics of apixaban, an oral, factor Xa inhibitor. Can J Clin Pharmacol. 2008; 15:e469. Abstract.

12. Mendell J, Tachibana M, Shi M, Kunitada S. Effects of food on the pharmacokinetics of edoxaban, an oral direct factor Xa inhibitor, in healthy volunteers. J Clin Pharmacol. 2011;51:687-694.

13. Furugohri T, Isobe K, Honda Y, et al. DU-176b, a potent and orally active factor Xa inhibitor: in vitro and in vivo pharmacological profiles. J Thromb Haemost. 2008;6(9):1542-1549.

14. Camm AJ, Bounameaux H. Edoxaban: a new oral direct factor xa inhibitor. Drugs. 2011;71(12):1503-1526.

15. Gulseth MP, Michaud J, Nutescu EA. Rivaroxaban: an oral direct inhibitor of factor Xa. Am J Health Syst Pharm. 2008;65:1520-1529.

16. Bathala MS, Masumoto H, Oguma T, He L, Lowrie C, Mendell J. Pharmacokinetics, biotransformation, and mass balance of edoxaban, a selective, direct factor xa inhibitor, in humans. Drug Metab Dispos. 2012;40:2250-2255.

17. EINSTEIN Investigators, Bauersachs R, Berkowitz SD, et al. Oral rivaroxaban for symptomatic venous thromboembolism. $N$ Engl J Med. 2010;363(26):2499-2510. 
18. EINSTEIN-PE Investigators, Büller HR, Prins MH, et al. Oral rivaroxaban for the treatment of symptomatic pulmonary embolism. $N$ Engl J Med. 2012;366(14):1287-1297.

19. Agnelli G, Buller HR, Cohen A, et al. Oral apixaban for the treatment of acute venous thromboembolism. N Engl J Med. 2013;369(9): 799-808.

20. Hokusai-VTE Investigators, Büller HR, Décousus H. Edoxaban versus warfarin for the treatment of symptomatic venous thromboembolism. N Engl J Med. 2013;369(15):1406-1415.

21. Agnelli G, Buller HR, Cohen A, et al. Apixaban for extended treatment of venous thromboembolism. N Engl J Med. 2013;368:699-708.

22. Nutescu E, Chuatrisorn I, Hellenbart E. Drug and dietary interactions of warfarin and novel oral anticoagulants: an update. J Thromb Thrombolysis. 2011;31:326-343.

23. Frost C, Wang J, Nepal S, et al. Effect of ketoconazole and diltiazem on the pharmacokinetics of apixaban, an oral direct factor Xa inhibitor. J Clin Pharmacol. 2009;49:1091-1130. Abstract.

24. Mendell J, Zahir H, Matsushima N, et al. Drug-drug interaction studies of cardiovascular drugs involving P-glycoprotein, an efflux transporter, on the pharmacokinetics of edoxaban, an oral factor Xa inhibitor. Am J Cardiovasc Drugs. 2013;13:331-342.

25. RuffCT, Giugliano RP, Antman EM, et al. Evaluation of the novel factor Xa inhibitor edoxaban compared with warfarin in patients with atrial fibrillation: design and rationale for the Effective aNticoaGulation with factor xA next GEneration in Atrial Fibrillation-Thrombolysis in Myocardial Infarction study 48 (ENGAGE AF-TIMI 48). Am Heart J. 2010;160:635-641.
26. Gruber A, Marzec UM, Buetehorn U, Hanson S, Perzborn E. Potential of activated prothrombin complex concentrate and activated factor VII to reverse the anticoagulant effects of rivaroxaban in primates. Blood. 2008;112:1307(abstract 3825).

27. Eerenberg ES, Kamphuisen PW, Sijpkens MK, Meijers JC, Buller HR, Levi M. Reversal of rivaroxaban and dabigatran by prothrombin complex concentrate: a randomized, placebo-controlled, crossover study in health subjects. Circulation. 2011;124:1573-1579.

28. Daiichi Sankyo Inc. A Two-part Study in Edoxaban-treated Healthy Subjects to a Punch Biopsy Bleeding Model Establish and to Evaluate the Effect of a 4-factor Prothrombin Complex Concentrate on Anticoagulation. Available from: http://clinicaltrials.gov/ct2/show/ NCT02047565?term=NCT02047565\&rank=1. NLM identifier: NCT02047565. Accessed July 12, 2014.

29. Andexanet alfa: FXa Inhibitor Antidote [webpage on the Internet]. South San Francisco: Portola Pharmaceuticals Inc. http://www.portola.com/ clinical-development/andexanet-alfa-prt4445-fxa-inhibitor-antidote/. Accessed June 2, 2014.

30. Lu G, DeGuzman FR, Lakhotia S, Hollenback SJ, Phillips DR, Sinha U. Recombinant antidote for reversal of anticoagulation by factor Xa inhibitors. Blood. 2008;112:abstract 983.

31. Mantha S, Ansell J. Indirect comparison of dabigatran, rivaroxaban, apixaban and edoxaban for the treatment of acute venous thromboembolism. J Thromb Thrombolys. Epub July 3, 2014.
Vascular Health and Risk Management

\section{Publish your work in this journal}

Vascular Health and Risk Management is an international, peerreviewed journal of therapeutics and risk management, focusing on concise rapid reporting of clinical studies on the processes involved in the maintenance of vascular health; the monitoring, prevention and treatment of vascular disease and its sequelae; and the involvement of

\section{Dovepress}

metabolic disorders, particularly diabetes. This journal is indexed on PubMed Central and MedLine. The manuscript management system is completely online and includes a very quick and fair peer-review system, which is all easy to use. Visit http://www.dovepress.com/ testimonials.php to read real quotes from published authors. 